\title{
Sleep and Quality of Life in Children with Cerebral Palsy
}

\author{
Danielle E. Sandella, B.S. ${ }^{1}$, Louise M. O’Brien, Ph.D. ${ }^{2}$, Laura K. Shank, Psy.D. ${ }^{1}$, and Seth A. \\ Warschausky, Ph.D. ${ }^{1,{ }^{*}}$ \\ ${ }^{1}$ Department of Physical Medicine and Rehabilitation, University of Michigan \\ 2 Sleep Disorders Center, Department of Neurology and Department of Oral \& Maxillofacial \\ Surgery, University of Michigan
}

\begin{abstract}
Objective-To examine the associations between sleep problems and quality of life (QoL) in children with cerebral palsy $(\mathrm{CP})$.

Methods-Prospective correlational study using parent-report forms to measure QoL and sleep disorder symptoms. Two groups comprised of 41 children with CP and 91 typically developing (TD) controls age 8-12 years participated in a prospective correlational study.

Results-Measures were the PedsQL-4.0 Generic scales and the Pediatric Sleep Questionnaire, with subscales of interest including sleep disordered breathing (SDB), excessive daytime sleepiness (EDS), insomnia (INS) and snoring (SNOR). Hierarchical regression analyses indicated that EDS contributed unique variance in physical QoL, and INS contributed unique variance in psychosocial QoL in children with CP; for TD children, sleep disorder symptoms were infrequent and not associated with physical or psychosocial QoL.
\end{abstract}

Conclusions-Findings highlight the importance of assessing sleep difficulties when addressing the needs of children with $\mathrm{CP}$.

\section{Keywords}

Sleep; Cerebral Palsy; Quality of Life; Sleep Disordered Breathing; Daytime Sleepiness; Child

\section{INTRODUCTION}

Sleep disorders are often reported by parents and may occur in approximately $25 \%$ of typically developing children; however, children with cerebral palsy (CP) are at an increased risk for sleep disorders [1,2]. In children with $\mathrm{CP}$, there is higher prevalence of initiation and maintenance of sleep disorders, sleep-wake transition disorders and excessive sleepiness and arousal disorders. Active epilepsy in children with $\mathrm{CP}$ has been found to be the strongest correlate of total sleep disturbance and, specifically, with excessive daytime sleepiness [2]. Total body involvement, including spastic quadriplegia and dystonic/dyskinetic subtypes, is strongly associated with disorders of initiation and maintenance of sleep.

Socioenvironmental factors such as family configuration and socioeconomic status also

Contact: Seth Warschausky, Ph.D., Department of Physical Medicine and Rehabilitation; University of Michigan, 325 E. Eisenhower Pkwy, Suite 300, Ann Arbor, MI, 48108-5744; sethaw@umich.edu; Tel. (734) 936-7051; Fax (734) 936-7048.

Conflict of Interest: None

Publisher's Disclaimer: This is a PDF file of an unedited manuscript that has been accepted for publication. As a service to our customers we are providing this early version of the manuscript. The manuscript will undergo copyediting, typesetting, and review of the resulting proof before it is published in its final citable form. Please note that during the production process errors may be discovered which could affect the content, and all legal disclaimers that apply to the journal pertain. 
appear to influence risk in this population, with single-parent families reporting higher prevalence of sleep-disordered breathing (SDB) [3]. Preterm gestation, a perinatal risk factor for $\mathrm{CP}$, is associated with lower sleep quality and duration, but the findings regarding low birth weight are less clear cut [4].

Excessive daytime sleepiness (EDS)—often a consequence of SDB and otherwise interrupted sleep - has been posited as an important symptom in relation to quality of life (QoL). No previous research has looked specifically at daytime sleepiness symptoms as a predictor of QoL [5]. Previous studies of relations between sleep disorders and QoL have largely focused on SDB and its treatment in otherwise typically developing children. Adenotonsillectomy, the first line treatment for SDB in children,as well as continuous positive airway pressure may lead to improvements in QoL dimensions for both children and their caregivers [6,7]. In their review of the literature on sleep disorders and QoL, Reimer and Flemons [8] discussed the need for further research that broadens the definition of sleep disorder to include conditions other than apnea, as well as the need for more specific QoL categories.

There is a paucity of literature that focuses on sleep disorders and CP, including a lack of prevalence estimates of SDB in CP. In the single previous study looking at SDB treatment and QoL in the CP population, the results indicate that treatment of SDB may have a positive effect on QoL. The study is limited, however, by a small sample size (19 participants), the exclusive use of children with Gross Motor Functional Classification System (GMFCS) [9] level V, and the retrospective nature of the study, requiring parents to recall aspects of the child's QoL up to three years prior to participation [7].

The study of children's QoL with CP is complicated by the heterogeneity of physical, cognitive, and sensory impairments associated with the condition [10]. Methodological differences include differing emphases on health status in the measurement of QoL and differing choice of informant, with most studies of grade school age children utilizing parent proxy ratings. There are inconsistent findings regarding differences in QoL between children with $\mathrm{CP}$ and typically developing peers. A number of studies have not found significant differences in QoL between the two groups [11,12]. Dickinson et al. [13] found that while overall QoL did not differ between the two groups, specific impairments and comorbidities, including pain, explained a small amount of variance in specific QoL domains. However, Varni et al. [14] found significant group differences in QoL, with children with CP reporting lower levels of physical, emotional, social, and school functioning than typically developing peers. In a study looking at predictors of QoL in children with CP, Soyupek et al. [15] found that incontinence, self-concept ratings, and GMFCS level were significant predictors of QoL. There are limited findings regarding $\mathrm{CP}$ comorbidities (including sleep disorders) as predictors of QoL or as moderators of the association between CP and QoL.

Children with $\mathrm{CP}$ are at risk for sleep disorders and decreased QoL. There is strong empirical evidence of an association between quality of sleep and QoL in general and small but consistent literature on the adverse effect of SDB on QoL. But there is minimal research that examines these associations in children with CP. We hypothesized that the presence of sleep problems would be associated with impaired QoL in children with CP.

\section{METHODS}

Participants

Two groups of participants included 41 children with congenital CP and 91 typically developing (TD) peers, ages 8-12 years (Table 1). Data were collected as part of a larger study of accessible neuropsychological procedures for children with CP. Inclusion criteria 
for the group with $\mathrm{CP}$ in that larger study included ages 8-16 years, ability to make a reliable dichotomous choice with a raw score of 12 or better on the Dichotomous Choice Screen [16]. The subset of children, ages 8-12 years, with complete data from the Pediatric Sleep Questionnaire (PSQ) [17] and the PedsQL were eligible for inclusion in this study. Children were excluded if they had a change in medications within the past month that could affect sleep such as anticonvulsant medications, sedatives, and neurostimulant medications. Exclusion criteria also included history of an acquired brain injury or other major neurological or psychiatric condition (for children with $\mathrm{CP}$, this refers to events subsequent to the onset and diagnosis of $\mathrm{CP}$ ) or inability of the parent or guardian to provide demographic and medical information.

In the group with $\mathrm{CP}, 88.5 \%$ of the sample exhibited spasticity as a primary tone abnormality. Of the total sample, $39 \%$ were reported as having spastic hemiplegia, $46.4 \%$ spastic diplegia, and $7.3 \%$ ataxia. Specific diagnosis type was missing in three cases. Functional levels using the GMFCS criteria were as follows: Level I 51.2\%, Level II 7.3\%, Level III 19.5\%, Level IV 7.3\%, Level V 4.9\%, and 9.8\% were missing data. Manual Ability Classification System (MACS) [18] levels included Level I 43.9\%, Level II 29.3\%, Level III 7.3\%, Level IV 4.9\%, Level V 2.4\%, and $12.2 \%$ were missing data. There was history of premature birth in $83.8 \%, 48.6 \%$ with very low birth weight $(<1500 \mathrm{~g})$ and seizures in $22.5 \%$ of the group with $\mathrm{CP}$.

As summarized in Table 1, group differences in age, socioeconomic status and gender distribution were not significant. There were statistically significant group differences in receptive vocabulary, $F(1,127)=9.9, p<.01, \eta^{2}=.07$, gestation, $F(1,91)=56.3, p<.01$, $\eta^{2}=.39$, seizure history, $\chi^{2}(1, \mathrm{~N}=40)=21.99, \mathrm{p}<.01$, and birth weight, $F(1,99)=75.5$, $p<.01, \eta^{2}=.44$.

\section{Instruments}

Parents completed the PSQ, a valid and reliable scale used to assess risk for sleep disturbance. The PSQ contains 48 question-items with a - yes/no/don't know response format. These items ask about obstructive sleep apnea symptoms such as snoring frequency, loud snoring, observed apneas, difficulty breathing during sleep, and daytime sleepiness. Subscales include the 22-item Sleep-Related Breathing Disorder scale (SRBD), a validated measure of sleep disordered breathing (SDB). This 22-item scale includes 6 behavioral items that were omitted in the current study to avoid artificial associations with behavior; thus, the 16-item version of the SRBD scale was used [19]. The mean response on non-missing items is the total score. A threshold of 0.33 , indicating that $33 \%$ of symptom-items are positive, is considered a positive screen for pediatric SDB. In addition, the PSQ includes the only pediatric behavioral rating scale for daytime sleepiness validated against sleep laboratory tests [20] and includes the following questions: Does your child 1) wake up feeling unrefreshed in the morning?; 2) have a problem with sleepiness during the day?; 3) Has a teacher or other supervisor commented that your child appears sleepy during the day?; 4) Is it hard to wake your child up in the morning?. Question items also query about insomnia (INS), snoring, and periodic leg movements during sleep (PLMS). The PSQ shows good internal consistency and test-retest reliability [21]. This scale has been utilized in numerous clinical research studies [22-24]. Data suggest that the SRBD score may be as predictive as polysomnographic measures of neurobehavioral problems at baseline and their improvement after adenotonsillectomy for sleep-disordered breathing [25]. PSQ subscales are generally interpreted in a dichotomous manner; however, for the purposes of this study, EDS, INS, snoring, and PLMS were treated as continuous variables.

The Pediatric Quality of Life Inventory (PedsQL) 4.0 Generic Core Scales [26,27] are measures of health-related quality of life that integrate generic and disease-specific 
assessment approaches. The parent proxy PedsQL instruments have age-band specific versions. For the purposes of this study, the 8-12 year old version was utilized. The Generic Core Scale version has 23 likert items that provide a total score and four subscale scores: Physical, Emotional, Social and School Functioning. In addition, two broadband summary scores can be calculated using the Generic Core Scales: the Physical Health Summary Score and the Psychosocial Health Summary Score. Summary scores are derived from the sum of the items over the number of items answered in the physical subscale (Physical Summary Score), and the emotional, social, and school subscales (Psychosocial Summary Score). Subscale internal consistency is adequate (Cronbach's alpha > .70) for generic subscales for the normative sample as a whole, but borderline for the Social and School functioning subscales in the 8-12 year old version. Validity studies include evidence that Generic Core Scales differentiate health-related QoL between typically developing children and children with $\mathrm{CP}$, as well as evidence of subscale profile differences associated with different levels of gross motor function in children with CP [27]. For the purposes of this study, the Generic Core Scale Psychosocial and Physical broadband scales were used in analyses.

Peabody Picture Vocabulary Test- $3^{\text {rd }}$ Edition (PPVT-III) [28]: The PPVT-III is an individually administered test designed to measure single word receptive vocabulary. For each test item, examinees are shown a page with four black and white illustrations, and a target word is orally presented. The child must identify the picture that best describes the word. This test measures receptive rather than expressive language skills, and examinees may simply point to the appropriate picture rather than provide a verbal response. The PPVT-III has a test-retest reliability ranging from .91 to .94 (median=.92). The PPVT-III has excellent validity as demonstrated by its high correlations with other measures of verbal ability (K-BIT vocabulary, .81 and WISC-III VIQ composite, .91).

Functional mobility was measured using the GMFCS. The GMFCS assesses gross motor functioning and activity limitations with a five level ordinal scale. Level one is described as able to walk without restrictions, with limitations, if any, in more advanced gross motor skills only; level five is described as severely limited self-mobility even with the use of assistive technology (i.e. power wheelchair). Interrater reliability is 0.75 and content and predictive validity are well-demonstrated in child and adult populations $[9,29,30]$.

Following Institutional Review Board approval, participants were recruited through community flyers and websites connected with two Midwest medical rehabilitation centers over a period of approximately 2 years. Parents provided informed consent, and children provided witnessed assent, for voluntary participation in the research program and in accordance with Institutional Review Board guidelines.

\section{Data Analyses}

Initially, we examined group differences in sleep and QoL as a partial replication of recent findings $[2,13,14]$. Group differences in PSQ and PedsQL profiles were examined with ANOVAs. Bivariate correlations between PSQ and PedsQL variables by group were compared using $\mathrm{r}$ to $\mathrm{z}$ transformations. In the group with $\mathrm{CP}$, bivariate correlations between GMFCS, PSQ and PedsQL scores were examined.

Following regression diagnostics, multiple regression analyses were conducted to determine if quality of life (PedsQL Physical and Psychosocial scores) could be predicted by sleep disorder (SRBD, insomnia, excessive daytime sleepiness and snoring scores). Separate multiple regression analyses were conducted for each group. Collinearity diagnostics did not reveal any threat to the validity of these models (e.g., all variance inflation factors $<1.25$ in both groups). Within the group with $\mathrm{CP}$, analyses also were conducted to examine medical variables and functional status as a correlate of sleep disorder and QoL. In the group with 
CP, GMFCS level was entered at Step 1 and in Step 2 INS, EDS, and snoring was entered in hierarchical stepwise regressions. Proportions of variance explained by the regression models were categorized according to Murphy and Myors' [31] criteria, with $R^{2}$ values less than .10 classified as small, $R^{2}$ values between .10 and .25 as medium, and $R^{2}$ values greater than .25 as large.

\section{RESULTS}

Table 2 presents the mean performances of both groups of participants on the main variables of interest. Regarding sleep disorder variables, the group with CP had significantly higher SRBD scores than the TD group, $\left(F[1,131]=29.99, p<.001, \eta^{2}=0.19\right.$, including higher PLMS $F[1,131]=9.69, p<.01, \eta^{2}=0.07$, INS, $. F[1,131]=11.20, p<.001, \eta^{2}=0.08$ and EDS, $F[1,131]=20.11, p<.001, \eta^{2}=0.13$ ). Snoring scores were not significantly different between groups $F[1,131], p>.05, \eta^{2}=0.01$. As illustrated in Table 2 , there was a restriction in range of scores on the PSQ in the TD sample, i.e., few children in the TD group had reported sleep difficulties.

Regarding QoL, the group with CP had significantly lower PedsQL Physical scores, $F[1$, $131]=194.63, p<.001, \eta^{2}=0.60$, and Psychosocial scores, $F[1,131]=80.85, p<.001, \eta^{2}$ $=0.38$.

Bivariate correlations are presented in Table 3. Fisher $\mathrm{r}$ to $\mathrm{z}$ transformations indicate that group differences are significant only in the correlations between INS and PedsQL Physical scores and, using a bonferroni correction, none of the group differences in bivariate correlations are significant.

In the group with $\mathrm{CP}$, bivariate correlations between gestational age, birth weight, and outcome variables were not significant. Within the group, there were no significant differences in sleep and QoL scores in those with and without history of seizure. There was a statistically significant correlation between GMFCS and PedsQL Physical score, however, $r=-.66, p<.01$.

Two sets of multiple regression analyses, one with the TD group and the other with the group with $\mathrm{CP}$, were conducted to examine the extent to which QoL, assessed with the PedsQL, could be predicted by sleep variables including INS, EDS, and snoring. In the multiple regression to predict PedsQL Physical in the group with CP, GMFCS level was entered in Step 1 and INS, EDS and snoring entered in Step 2.

Regression models differed in the two groups. In the group with $\mathrm{CP}$, the multiple regression to predict PedsQL Psychosocial from EDS, INS and snoring scores was significant, accounting for a medium portion of the variance, $F(4,36)=3.92, p<.05, R^{2}=0.33$, and INS was a significant predictor. In the TD group, the regression was not significant, $F$ (3, $90)=2.26, p>.05, R^{2}=0.07$.

In the group with CP, the hierarchical regression to predict PedsQL Physical with GMFCS, EDS, INS and snoring scores was statistically significant, accounting for a large portion of the variance, $F(4,36)=11.20, p<.001, R^{2}=0.58$, with GMFCS and EDS contributing significant unique variance to the PedsQL Physical score (Table 5). In the TD group, a multiple regression to predict PedsQL Physical from EDS, INS and snoring was not significant, $F(3,90)=2.13, p>.05, R^{2}=0.07$. 


\section{DISCUSSION}

Results partially support previous findings of risks for sleep disruption and lower QoL in children with CP. Sleep difficulties in children with CP include greater frequencies of insomnia and excessive daytime sleepiness. There also were indications of greater periodic leg movement at night, though this finding may be confounded by the nature of motor abnormalities associated with CP. Findings also indicate lower parent-reported QoL in children with CP compared with TD peers. This lower QoL was noted in both physical and psychosocial domains, consistent with the Varni et al. ${ }^{14}$ findings.

There were significant group differences in the effects that sleep disturbances had on QoL. While GMFCS levels significantly predicted QoL in children with CP, INS and EDS contributed unique variance as well. In the group with CP, psychosocial QoL was affected by insomnia; EDS predicted lower physical QoL in the group. Effects of sleep variables on QoL of the TD group were not significant.

Functional ability (i.e., GMFCS level) is a known predictor of physical QoL and has been a focus for treatment in children with CP; however, it is possible that feeling sleepy during the day exacerbates existing physical impairments, requiring even more effort than usual for movement and resulting in a lower physical QoL as perceived by caregivers. The unique variance contributed by EDS, however, suggests that further investigation into sleep problems could potentially lead to qualitative improvements in QoL, addressing a comorbid risk that may not be addressed by focused treatment of motor impairments.

The composition of the group with CP, score distributions in the TD group and nature of study instrumentation affect the generalizability of findings. The group with $\mathrm{CP}$ was comprised largely of children who were higher functioning based on GMFCS, MACS, and PPVT-III. The survey instruments used to assess sleep and QoL, including the PSQ and the PedsQL, were parent reports, with no direct input from the children themselves. Parent- and self-report ratings on the PedsQL for children with CP have previously been compared and parent ratings, in general, are comparable to children's self-reports, although there are discrepancies primarily in the psychosocial domain [32]. As significant associations between INS and psychosocial QoL are one of the significant findings in the present study, future investigation is warranted in this area using child self-reports of QoL instead of or in addition to the parent-report forms.

To follow up on the identified associations between sleep and QoL in children with CP, specific scales to measure daytime sleepiness would be recommended. While the EDS subscale of the PSQ is a validated scale for evaluating EDS, it contains only four questions and may not be comprehensive. More detailed measurement may help to refine our understanding of the nature of EDS in children with CP.

In summary, this study examined between-group differences in the associations between sleep and QoL between children with CP and their typically developing peers. Insomnia, excessive daytime sleepiness, and functional motor abilities were associated with lower QoL in children with CP. Findings highlight the importance of considering sleep difficulties when addressing the needs of children with $\mathrm{CP}$.

\section{Acknowledgments}

This work was supported by NIH R21 HD052592-01A, NIH R21 HD057344-01, U.S. Department of Education, National Institute on Disability and Rehabilitation Research award FI H133G070044 and the Mildred Swanson Foundation. 


\section{References}

1. Owens J. Classification and epidemiology of childhood sleep disorders. Sleep Medicine Clinics. 2007; 2:353.

2. Newman C, O'Regan M, Hensey O. Sleep disorders in children with cerebral palsy. Dev Med Child Neurol. 2006; 48:564-8. [PubMed: 16780625]

3. Rosen C. Prevalence and risk factors for sleep-disordered breathing in 8- to 11-year-old children: association with race and prematurity*. J Pediatr. 2003; 142:383. [PubMed: 12712055]

4. Strang-Karlsson S, Raikkonen K, Kajantie E, et al. Sleep Quality in Young Adults with Very Low Birth Weight--the Helsinki Study of Very Low Birth Weight Adults. J Pediatr Psychol. 2007; 33:387. [PubMed: 18077474]

5. Flemons WW, Tsai W. Quality of life consequences of sleep-disordered breathing. J Allergy Clin Immunol. 1997; 99:S750-S6. [PubMed: 9042067]

6. de Serres LM, Derkay C, Sie K, et al. Impact of Adenotonsillectomy on Quality of Life in Children With Obstructive Sleep Disorders. Arch Otolaryngol Head Neck Surg. 2002; 128:489-96. [PubMed: 12003578]

7. Hsiao KH, Nixon GM. The effect of treatment of obstructive sleep apnea on quality of life in children with cerebral palsy. Res Dev Disabil. 2008; 29:133-40. [PubMed: 17331700]

8. Reimer MA, Flemons WW. Quality of life in sleep disorders. Sleep Med Rev. 2003; 7:335-49. [PubMed: 14505600]

9. Palisano R, Rosenbaum P, Walter S, Russell D, Wood E, Galuppi B. Development and reliability of a system to classify gross motor function in children with cerebral palsy. Dev Med Child Neurol. 1997; 39:214-23. [PubMed: 9183258]

10. Livingston MH, Rosenbaum PL, Russell DJ, Palisano RJ. Quality of life among adolescents with cerebral palsy: what does the literature tell us? Dev Med Child Neurol. 2007; 49:225-31. [PubMed: 17355481]

11. Chow SM, Lo SK, Cummins RA. Self-perceived quality of life of children and adolescents with physical disabilities in Hong Kong. Qual Life Res. 2005; 14:415-23. [PubMed: 15892430]

12. Vinson J, Shank L, Thomas P, Warschausky S. Self-generated Domains of Quality of Life in Children with and Without Cerebral Palsy. J Dev Phys Disabil. 2010 published online 2010 Jan 28.

13. Dickinson HO, Parkinson KN, Ravens-Sieberer U, et al. Self-reported quality of life of 8-12-yearold children with cerebral palsy: a cross- sectional European study. Lancet. 2007; 369:2171-8. [PubMed: 17604799]

14. Varni J, Burwinkle T, Sherman S, et al. Health-related quality of life of children and adolescents with cerebral palsy: hearing the voices of the children. Dev Med Child Neurol. 2005; 47:592-7. [PubMed: 16138665]

15. Soyupek F, Aktepe E, Savas S, Askin A. Do the self-concept and quality of life decrease in CP patients? Focussing on the predictors of self- concept and quality of life. Disabil Rehabil. 2010; $0: 1-7$.

16. Van Tubbergen M, Warschausky S, Birnholz J, Baker S. Choice beyond preference: Conceptualization and assessment of choice-making skills in children with significant impairments. Rehabil Psychol. 2008; 53:93-100.

17. Chervin R, Hedger K, Dillon J, Pituch K. Pediatric sleep questionnaire (PSQ): validity and reliability of scales for sleep-disordered breathing, snoring, sleepiness, and behavioral problems. Sleep Med. 2000; 1:21-32. [PubMed: 10733617]

18. Eliasson AC, Krumlinde-Sundholm L, Rösblad B, et al. The Manual Ability Classification System (MACS) for children with cerebral palsy: scale development and evidence of validity and reliability. Dev Med Child Neurol. 2006; 48:549. [PubMed: 16780622]

19. Chervin RD, Archbold KH, Dillon JE, et al. Inattention, Hyperactivity, and Symptoms of SleepDisordered Breathing. Pediatrics. 2002; 109:449-56. [PubMed: 11875140]

20. Chervin RD. Subjective sleepiness and polysomnographic correlates in children scheduled for adenotonsillectomy vs. other surgical care. Sleep. 2006; 29:495. [PubMed: 16676783] 
21. Guilleminault C, Lin CM, Gonçalves MA, Ramos E. A prospective study of nocturia and the quality of life of elderly patients with obstructive sleep apnea or sleep onset insomnia. $\mathbf{J}$ Psychosom Res. 2004; 56:511-5. [PubMed: 15172207]

22. Guilleminault C, Lee JH, Chan A, Lopes M, Huang Y, da Rosa A. Non- REM-sleep instability in recurrent sleepwalking in pre-pubertal children. Sleep Med. 2005; 6:515-21. [PubMed: 15994122]

23. Huang Y, White D, Malhotra A. The Impact of Anatomic Manipulations on Pharyngeal Collapse*. Chest. 2005; 128:1324-30. [PubMed: 16162725]

24. Chervin RD, Weatherly RA, Garetz SL, et al. Pediatric Sleep Questionnaire: Prediction of Sleep Apnea and Outcomes. Arch Otolaryngol Head Neck Surg. 2007; 133:216-22. [PubMed: 17372077]

25. Varni J, Seid M, Kurtin P. PedsQL 4.0: reliability and validity of the Pediatric Quality of Life Inventory version 4.0 generic core scales in healthy and patient populations. Med Care. 2001; 39:800-12. [PubMed: 11468499]

26. Varni JW, Burwinkle TM, Berrin SJ, et al. The PedsQL in pediatric cerebral palsy: reliability, validity, and sensitivity of the Generic Core Scales and Cerebral Palsy Module. Dev Med Child Neurol. 2006; 48:442-9. [PubMed: 16700934]

27. Dunn, LDLM. Examiner's Manual for the PPVT-III Peabody Picture Vocabulary Test. Circle Pines, MN: American Guidance Service; 1997.

28. Sandström K, Alinder J, Oberg B. Descriptions of functioning and health and relations to a gross motor classification in adults with cerebral palsy. Disabil Rehabil. 2004; 26:1023. [PubMed: 15371039]

29. Wood E, Rosenbaum P. The gross motor function classification system for cerebral palsy: a study of reliability and stability over time. Dev Med Child Neurol. 2000; 42:292. [PubMed: 10855648]

30. Murphy, KR.; Myors, B. Statistical Power Analysis. Routledge: 2004.

31. Majnemer A, Shevell M, Law M, Poulin C, Rosenbaum P. Reliability in the ratings of quality of life between parents and their children of school age with cerebral palsy. Qual Life Res. 2008;

17:1163. [PubMed: 18821030] 


\section{Table 1}

Medical and Demographic Characteristics by Group

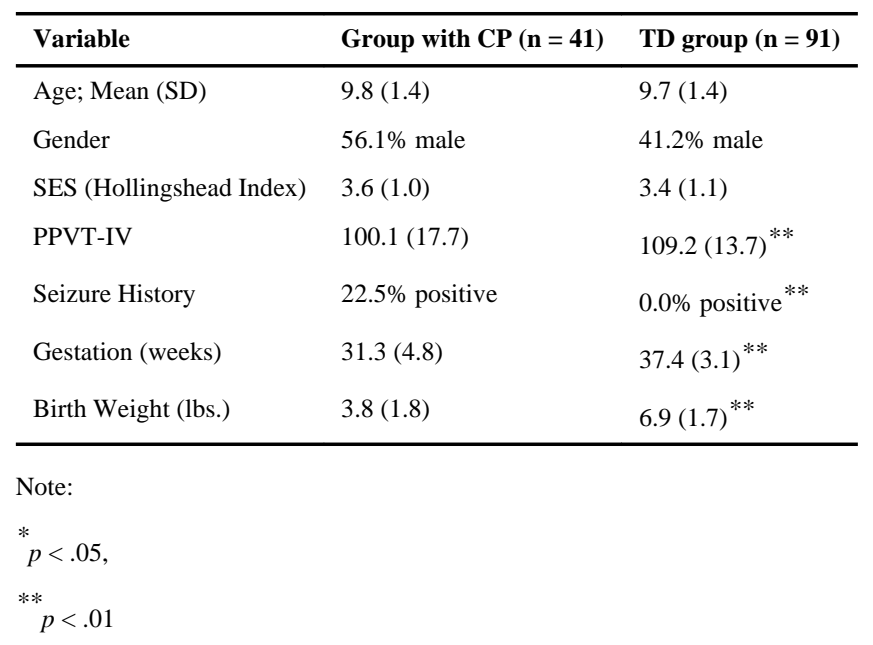


Table 2

Means and Standard Deviations of Study Variables by Group

\begin{tabular}{lll}
\hline Variable & Group with CP $(\mathbf{n}=\mathbf{4 1})$ & TD group $(\mathbf{n}=\mathbf{9 1})$ \\
\hline SRBD; Mean (SD) & $2.5(1.8)$ & $1.0(1.2)^{* *}$ \\
SDB & $7.3 \%$ positive & $2.2 \%$ positive \\
PLMS & $.10(.30)$ & $0.0(0.0)^{*}$ \\
INS & $.56(.87)$ & $.19(.42)^{*}$ \\
EDS & $1.0(1.1)$ & $.33(.63)^{* *}$ \\
SNOR & $.49(.78)$ & $.30(.72)$ \\
PedsQL Psychosocial & $64.0(19.1)$ & $88.3(11.7)^{* *}$ \\
PedsQL Physical & $44.1(33.4)$ & $96.9(9.3)^{* *}$ \\
\hline
\end{tabular}

Note: $\mathrm{SRBD}=$ Sleep-Related Breathing Disturbance scale; $\mathrm{SDB}=$ sleep-disordered breathing screen; PLMS = periodic leg movements during sleep; INS = insomnia; EDS = excessive daytime sleepiness; SNOR = snoring; PedsQL Psychosocial = PedsQL psychosocial health summary score; PedsQL Physical $=$ PedsQL physical health summary score.

* $p<.05$,

$* * x<.01$ 


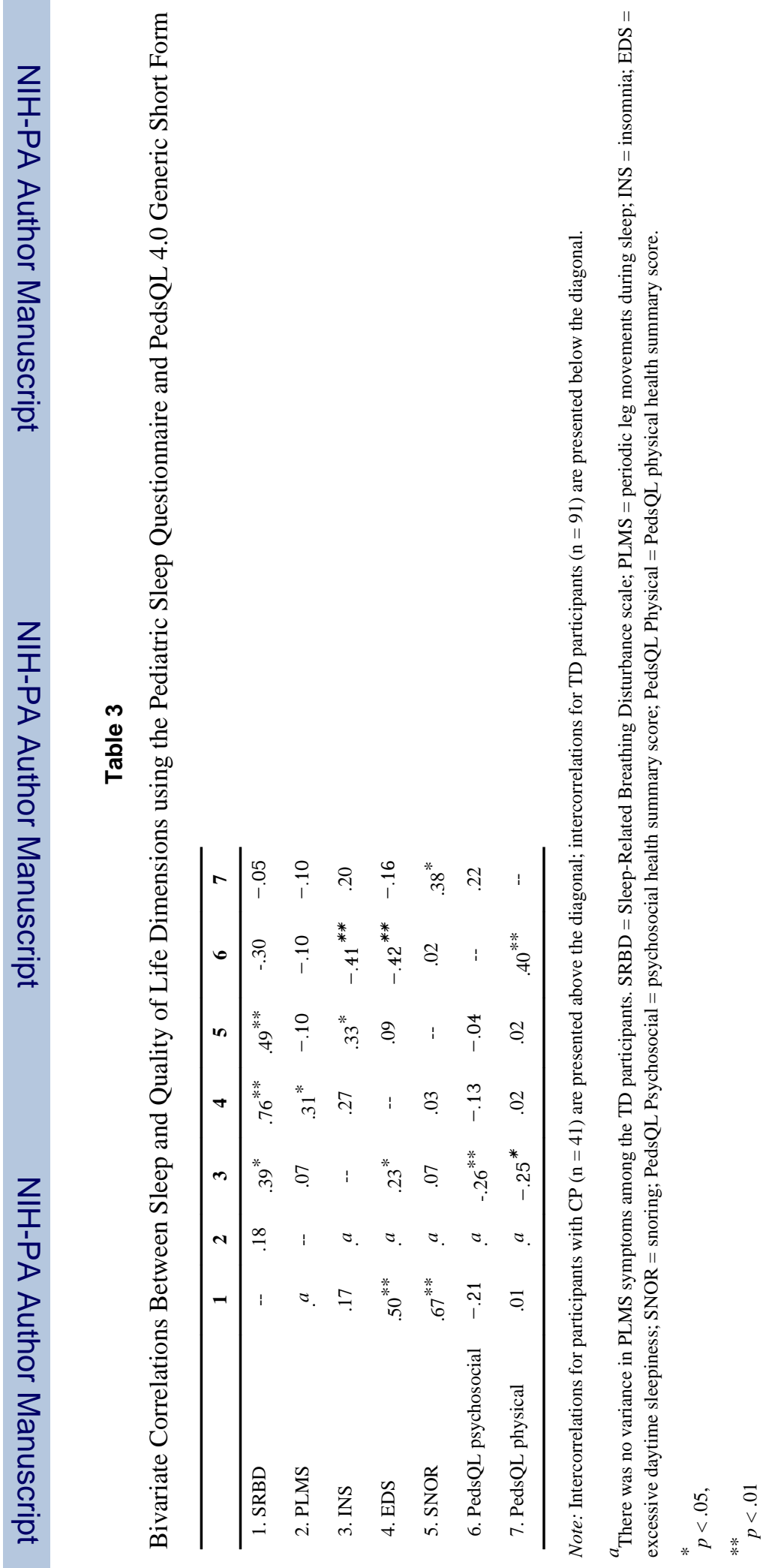




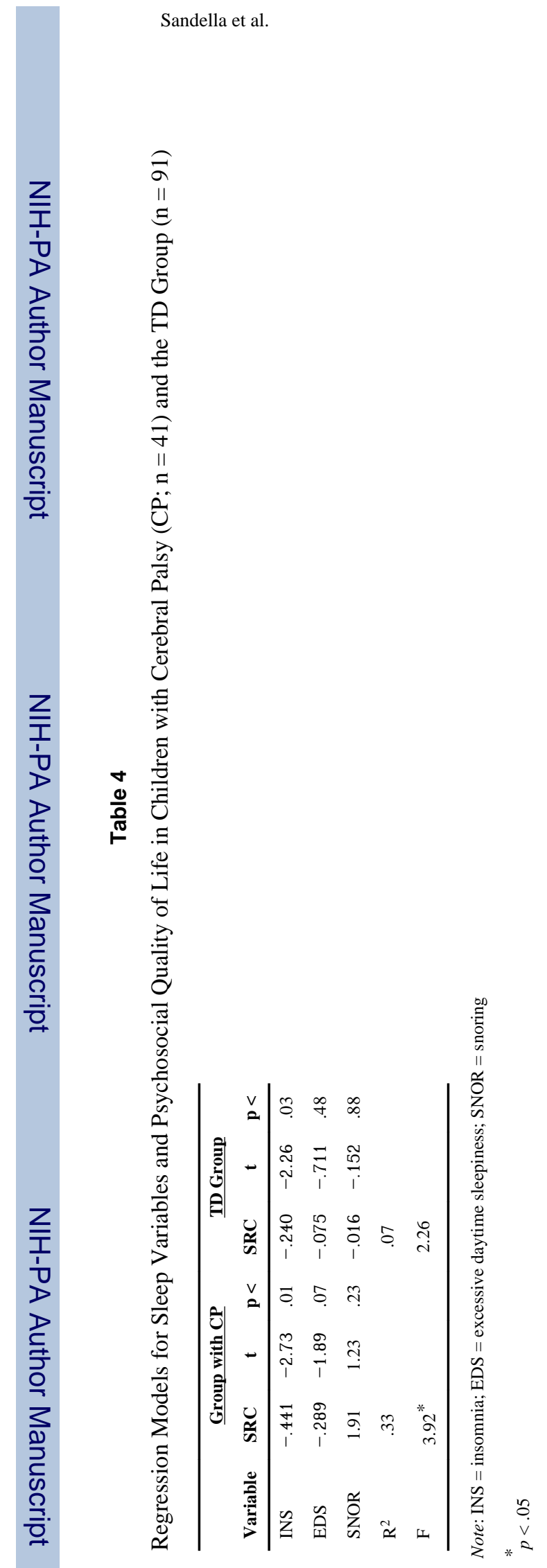

Sleep Med. Author manuscript; available in PMC 2012 March 1. 


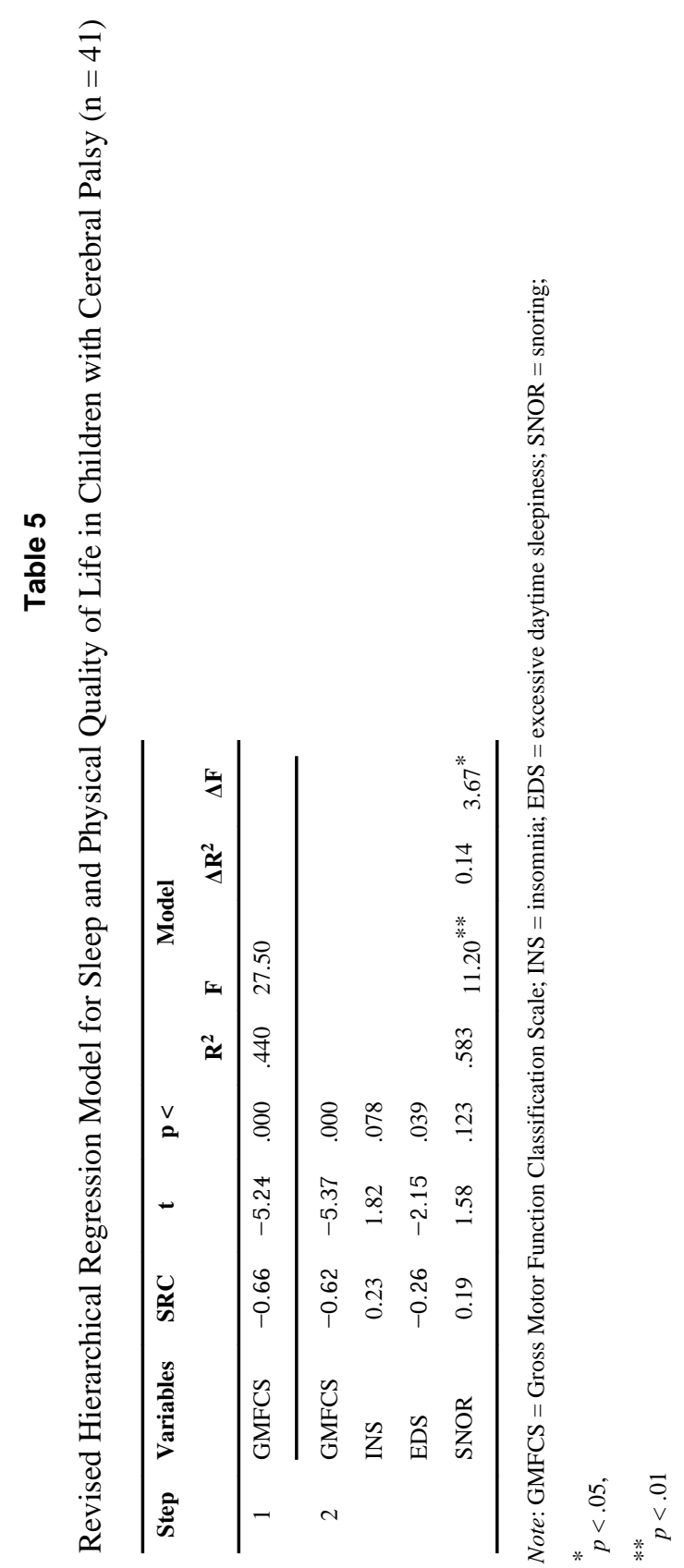

Sleep Med. Author manuscript; available in PMC 2012 March 1. 